\title{
ON $O_{\omega}$-IDEALS IN $C(X)$
}

J. G. HORNE, JR.

1. Introduction. The category of ideals mentioned in the title may be regarded, on the one hand, as a natural generalization of Milgram's notion of $O$-ideal [10] and, on the other, as another specialization of the notion of $R$-ideal [7]. Specifically, an $O_{\omega}$-ideal of $C(X)$ is an ideal $J$ which satisfies the following condition: $f_{1}, f_{2} \in J$ implies the existence of elements $e \in J$ and $h_{1}, h_{2} \in C(X)$ such that $f_{i}=h_{i} e$, for $i=1,2$. We show that any ideal which is an intersection of prime ideals is an $O_{\omega}$-ideal. Some results follow concerning the algebraic and topological properties of such ideals. In the final section, we use some results from [7] to obtain a necessary and sufficient condition that the intersection of a family of prime ideals be an $O$-ideal.

$\$ 2$ contains definitions and results to be used later, but in a much more general form than necessary for the remainder of the paper. This generality is consistent, however, with our continued interest in the notion of $R$-ideal.

2. Preliminaries. Let $S$ be a set. By a proper subset of $S$ is meant a nonempty set $J \subset S$ such that $J \neq S$. We review briefly the definitions of two familiar topologies which may be imposed on any family $\mathfrak{S}$ of proper subsets of $S$. For a subset $\mathfrak{A}$ of $\mathfrak{S}$, let us denote the set $\cup\{J: J \in \mathfrak{A}\}$ more briefly by $\cup \mathfrak{A}$ and the set $\cap\{J: J \in \mathfrak{A}\}$ by $\cap \mathfrak{A}$. Set $c_{1}(\mathfrak{H})=\{J \in \mathfrak{S}: J \subset \cup \mathfrak{H}\}$ and $c_{2}(\mathfrak{H})=\{J \in \mathfrak{S}: J \supset \cap \mathfrak{U}\}$. Now let $\mathfrak{F}_{i}$ denote the family of all sets $\mathfrak{A} \subset \mathfrak{S}$ such that $c_{i}(\mathfrak{H})=\mathfrak{A}$, for $i=1,2$. The dual Stone [Stone] topology is the smallest topology $\Delta[\Sigma]$ on $\subseteq$ whose family of closed sets includes $\mathfrak{F}_{1}\left[\mathfrak{F}_{2}\right]$. The resulting topological space is designated by $\mathfrak{S}_{\Delta}\left[\mathfrak{S}_{\Sigma}\right]$. It is known that $c_{1}\left[c_{2}\right]$ is the closure operation for $\Delta[\Sigma]$ if $c_{1}(\mathfrak{A} \cup \mathfrak{B}) \subset c_{1}(\mathfrak{A}) \cup c_{1}(\mathfrak{B})\left[c_{2}(\mathfrak{H} \cup \mathfrak{B}) \subset c_{2}(\mathfrak{H}) \cup c_{2}(\mathfrak{B})\right]$, for every pair of sets $\mathfrak{A}, \mathfrak{B C} \subseteq$ (see [1], for example). When this inclusion holds, it is said that $\subseteq$ admits the dual Stone [Stone] topology. ${ }^{1}$ Our usage of such terms as " $\Delta$-closure" and " $\Sigma$-closed" agrees with [8]. A fact which we use several times without further mention is that if $\mathfrak{I} \subset \mathfrak{S}$, then the topology induced on $\mathfrak{I}$ by $\mathfrak{S}_{\Delta}\left[\mathfrak{S}_{\Sigma}\right]$ is the same as that of $\mathfrak{I}_{\Delta}\left[\mathfrak{I}_{\Sigma}\right]$. Thus, if $\mathfrak{S}$ admits the dual Stone or the Stone topology, so does every subset of $\mathfrak{S}$.

Presented to the Society August 30, 1957; received by the editors September 26, 1957.

${ }^{1}$ In the sense of [7], this is the same as saying that the dual Stone [Stone] paratopology is a topology. 
Now suppose that $S$ is endowed with a binary relation $R$. We recall the following from [7].

Definition 1. A nonempty set $I C S$ is an $R$-ideal provided (i) $f \in I$, $h \in S$ and $h R f$ imply $h \in I$, and (ii) $f_{1}, f_{2} \in I$ imply there exists $e \in I$ such that $f_{i} R e$, for $i=1,2$.

A subset which satisfies condition (i) of the definition is called an ideal with respect to $R$. A subset satisfying condition (ii) is $R$-directed.

Analogues of Lemma 2.2 and Theorem 3.10 of [7] hold for the family $\subseteq$ of all proper $R$-ideals of $S$. Proofs of these results are easy to construct, or may be patterned after the proofs of the corresponding results just mentioned, and are therefore omitted.

Lemma 1. If $J$ is an $R$-ideal and $J_{1}$ and $J_{2}$ are ideals with respect to $R$ such that $J \subset J_{1} \cup J_{2}$, then $J \subset J_{1}$ or $J \subset J_{2}$.

THEOREM 1. The family of all proper R-ideals of a set $S$ admits the dual Stone topology.

Now assume that $S$ is a commutative semigroup with identity. That is, $S$ is endowed with a binary associative multiplication such that $f g=g f$ for all $f, g \in S$, and $S$ contains a (unique) element 1 such that $1 f=f$ for all $f \in S$. For $A, B \subset S$ we let $A B$ denote, as usual, the set $\{a b: a \in A$ and $b \in B\}$. A subset $J \subset S$ is a semigroup ideal (abbreviated s.g. ideal) if $S J \subset J$. A s.g. ideal $P$ is a prime s.g. ideal provided $(S \backslash P)(S \backslash P) \subset S \backslash P$. A principal s.g. ideal is a s.g. ideal $J$ generated by a single element; i.e., for some $f \in S, J=[f]$, where $[f]$ denotes the set $f S$.

Several relations on $S$ are of interest here. The canonical order on $S$ is the relation $O=\{(f, e): f e=f\}$. The resulting concept of $O$-ideal yields an extension of Milgram's notion [10]. The relation which is of chief concern in this paper is following natural extension of the canonical order.

Definition 2. The weak canonical order on $S$ is the relation $O_{\omega}$ $=\{(f, e): f \in e S\}$.

Of brief interest is the inverse $O_{\omega}^{-1}$ of the weak canonical order: $O_{\omega}^{-1}=\{(f, e): e \in f S\}$.

For a fixed $f \in S$, the set of $h$ such that $h O_{\omega} f$ can be described in two ways: By the definition of $O_{\omega}^{-1}$, it is the same as the set of $h$ such that $f O_{\omega}^{-1} h$. On the other hand, it also coincides with the principal ideal $[f]$. Hence, a set $J \subset S$ is a s.g. ideal if and only if $J$ is an ideal with respect to $O_{\omega}$, and if and only if $S \backslash J$ is an ideal with respect to $O_{\omega}^{-1}$.

If $P$ is a prime s.g. ideal of $S$ and $f, g \notin P$, then $f g \notin P$. Since $f O_{\omega}^{-1} f g$ 
and $g O_{\omega}^{-1} f g$, it follows that $S \backslash P$ is $O_{\omega}^{-1}$-directed. According to the above observations, we have the result:

(1) If $P$ is a prime s.g. ideal of $S$, then $S \backslash P$ is an $O_{\omega}^{-1}$-ideal.

Now let $\mathfrak{B}$ denote the set of proper prime s.g. ideals of $S$, and let $\mathfrak{P}^{\prime}$ denote the set of complements of members of $\mathfrak{B}$. The correspondence $P \rightarrow S \backslash P$ obviously induces a homeomorphism from $\mathfrak{P}_{\Sigma}$ to $\mathfrak{B}_{\Delta}^{\prime}$. Hence, as a corollary to Theorem 1 , we have the essentially known result $[2]$ :

CoROllary 1.1. The collection of proper prime s.g. ideals of $S$ admits the Stone topology.

The following characterization of $O_{\omega}$-ideals in $S$ is immediate.

(2) A subset $J \subset S$ is an $O_{\omega}$-ideal if and only if (i) $J$ is a s.g. ideal and (ii) $f_{1}, f_{2} \in J$ imply there exist elements $e \in J$ and $h_{1}, h_{2} \in S$ such that $f_{i}=h_{i} e$, for $i=1,2$.

In particular, principal s.g. ideals and $O$-ideals are obviously $O_{\omega^{-}}$ ideals.

Now suppose $(R,+, \cdot)$ is a commutative ring with identity, and let $S$ denote its multiplicative semigroup. An ideal of $R$ is, as usual, a subset $J \subset R$ which is a s.g. ideal of $S$ such that $J+J \subset J$. A prime ideal is, of course, an ideal which is a prime s.g. ideal of $S$. For a finite set $f_{1}, f_{2}, \cdots, f_{n}$ of elements of $R$, we let $\left[f_{1}, f_{2}, \cdots, f_{n}\right]$ denote the smallest ideal of $R$ which contains the elements $f_{1}, f_{2}, \cdots, f_{n}$. Since a principal s.g. ideal of $S$ is an ideal of $R$, this notation creates no conflict with our earlier notation for principal s.g. ideals.

Finally, we list two useful results whose easy proofs are omitted.

(3) An $O_{\omega}$-ideal of $S$ is an ideal of $R$.

(4) In order that every ideal of $R$ be an $O_{\omega}$-ideal, it is necessary and sufficient that for every $f_{1}, f_{2} \in R$, the ideal $\left[f_{1}, f_{2}\right]$ is an $O_{\omega}$-ideal.

3. $O_{\omega}$-ideals in $C(X)$. Let $X$ be a topological space and let $C(X)$ denote the ring of all real-valued continuous functions on $X$ under ordinary pointwise addition and multiplication. For $f \in C(X),|f|$ denotes the member of $C(X)$ defined by the identity $|f|(x)=|f(x)|$.

THEOREM 2. If $J$ is an ideal of $C(X)$ which satisfies $(*) f \in J$ implies $|f|^{1 / 2} \in J$, then $J$ is an $O_{\omega}$-ideal.

Proof. We only have to show that $J$ is $O_{\omega}$-directed. Suppose $f_{1}, f_{2} \in J$. Let $e=\left(\left|f_{1}\right|+\left|f_{2}\right|\right)^{1 / 2}$. Then $e \in J$ by hypothesis (since in general, (*) implies that if $f \in J$ then $|f| \in J$ ). Now define

$$
\begin{array}{ll}
h_{i}(x)=f_{2}(x) / e(x) & \text { if } e(x) \neq 0, \text { and } \\
h_{i}(x)=0 & \text { if } e(x)=0, \text { for } i=1,2 .
\end{array}
$$


Then $h_{i} \in C(X)$; the details follow from the inequalities

$$
\left|h_{i}(x)\right|=\left|f_{i}(x)\right| /\left(\left|f_{1}(x)\right|+\left|f_{2}(x)\right|\right)^{1 / 2} \leqq\left|f_{i}(x)\right|^{1 / 2}
$$

which hold if $e(x) \neq 0$.

It is clear that $f_{i}=h_{i} e$, for $i=1,2$, so the proof of the theorem is complete.

REMARK 1. Recall that the $m$-topology on $C(X)$ is the topology having as a base for its neighborhoods of zero the sets $U_{\pi}=\{f \in C(X)$ : $|f|<\pi\}$ where $\pi$ is a strictly positive member of $C(X)[6 ; 5]$. The functions $h_{i}$ in the above theorem actually belong to the $m$-closure of $J$. For let $\pi$ be as above, and define $g_{i}=f_{i} /(e+\pi)$, for $i=1,2$. Then

$$
\left|g_{i}(x)-h_{i}(x)\right|<\pi(x) \quad \text { for all } x \in X .
$$

For if $e(x)=0$, then $f_{i}(x)=h_{i}(x)=0$, so the inequality is immediate. Suppose $e(x) \neq 0$. Then

$$
\left|g_{i}(x)-h_{i}(x)\right|=\left|f_{i}(x)\right| \pi(x) /\left(e(x)^{2}+e(x) \pi(x)\right) .
$$

Since

$$
\left|f_{i}(x)\right| \leqq\left(\left|f_{1}(x)\right|+\left|f_{2}(x)\right|\right)=e(x)^{2},
$$

we have

$$
\left|f_{i}(x)\right| \pi(x)<\left(e(x)^{2}+e(x) \pi(x)\right) \pi(x),
$$

which yields the desired inequality in this case also.

REMARK 2. It is easy to see that a prime ideal of $C(X)$ satisfies condition $(*)$ of the theorem. In particular, every maximal ideal of $C(X)$ is an $O_{\omega}$-ideal. Evidently, the intersection of ideals which satisfy $(*)$ also satisfies this condition. Hence, we have

CoROLlary 2.1. If $J$ is an intersection of prime ideals of $C(X)$, then $J$ is an $O_{\omega}$-ideal.

In virtue of [5, Theorem 9], the fact that maximal ideals are prime, and Remark 1 above, we also have

Corollary 2.2. If $J$ is an $m$-closed ideal, then $J$ is an $O_{\omega}$-ideal such that $J^{2}=J$.

To obtain the following corollary, we also use (3) above, of course.

Corollary 2.3. Suppose $X$ and $Y$ are topological spaces and $T: C(X) \rightarrow C(Y)$ is a multiplicative isomorphism onto. If $J$ has the property of being (i) an intersection of prime ideals, (ii) an m-closed ideal, or (iii) a maximal ideal of $C(X)$, then the image of $J$ is a (ring) ideal of $C(Y)$ with the corresponding property. 
By also making use of Theorem 1, we have

COROLlary 2.4. The family of all proper prime ideals (and therefore, the family of all maximal ideals) of $C(X)$ admits the dual Stone topology.

The following theorem indicates that in general, $C(X)$ contains ideals which are not $O_{\omega}$-ideals. Recall that an $F$-space is a space $X$ such that every finitely generated ideal of $C(X)$ is principal [4].

Theorem $3 .^{2}$ Every ideal of $C(X)$ is an $O_{\omega}$-ideal if and only if $X$ is an F-space.

Proof. Suppose $X$ is an $F$-space. Let $f_{1}, f_{2} \in C(X)$. The ideal $\left[f_{1}, f_{2}\right]$ is principal, and therefore is an $O_{\omega}$-ideal. Hence every ideal of $C(X)$ is an $O_{\omega}$-ideal, by (4) above.

Conversely, suppose every ideal of $C(X)$ is an $O_{\omega}$-ideal. Among several characterizations of $F$-spaces given in [4], one is that for every $f \in C(X), f$ belongs to $[|f|]$. Now by hypothesis, the ideal $[(f \vee 0),(f \wedge 0)]$ is an $O_{\omega}$-ideal (where " $\vee$ " and " $\wedge$ " denote the usual lattice operations on $C(X))$. Therefore, there exist elements $h, h^{\prime}, g$ and $g^{\prime} \in C(X)$ such that

$$
\begin{aligned}
& f \vee 0=h\left(g(f \vee 0)+g^{\prime}(f \wedge 0)\right), \text { and } \\
& f \wedge 0=h^{\prime}\left(g(f \vee 0)+g^{\prime}(f \wedge 0)\right) .
\end{aligned}
$$

If $f(x)>0$, we have $1=h(x) g(x)$ and $0=h^{\prime}(x) g(x)$, while if $f(x)<0$, we have $0=h(x) g^{\prime}(x)$ and $1=h^{\prime}(x) g^{\prime}(x)$. Hence, with $k=h g-h^{\prime} g^{\prime}$, we have $f=k|f| \in[|f|]$, and the proof of the theorem is complete.

4. A property of prime ideals in $C(X)$. It follows from [4, Theorem 1.4] and [7, Example 2.14] that every maximal $O$-ideal is an intersection of prime ideals. Actually, this is true of arbitrary $O$-ideals in a commutative ring $R$ with zero radical; (we say that $R$ has zero radical if, for $f \in R$ and $n$ a positive integer, $f^{n}=0$ implies $f=0$; for the definition of the radical of an ideal, see [9, p. 99]).

Theorem 4. Every O-ideal $I$ in a commutative ring $R$ with zero radical is an intersection of prime ideals.

Proof. Suppose $f \in R$ is an element and $n$ is a positive integer such that $f^{n} \in I$. There exists an element $e \in I$ such that $f^{n} e=f^{n}$, so $f^{n}(1-e)=0$. Therefore $(f(1-e))^{n}=0$. Since $R$ has zero radical, it follows that $f(1-e)=0$ and hence $f=f e \in I$. By Theorem 24 of [9], $I$, being its own radical, is an intersection of prime ideals.

2 This corrects Theorem 2 of the abstract of this paper. 
It is now natural to ask when the intersection of a family of prime ideals of $C(X)$ is actually an $O$-ideal.

Let $\mathfrak{B}$ denote the family of proper prime ideals and $\mathfrak{M}$ the family of maximal $O$-ideals of $C(X)$. If $f$ is a function on a set $S$ and $A \subset S$, we use $f[A]$ to denote the image, under $f$, of the set $A$. For $I \subset C(X)$, set $\mathfrak{B}(I)=\{P \in \mathfrak{B}: I \subset P\}$. According to Corollary $1.1, \mathfrak{B}$ admits the Stone topology, and according to Corollary 2.4, $\mathfrak{B}$ also admits the dual Stone topology. These topologies are not comparable if $C(X)$ contains a nonmaximal prime ideal $P$ (that is, if $X$ is not a $P$-space [3, Theorem 5.3]). For if $P$ is contained in the maximal ideal $N$, and $P \neq N$, then $\{N\}$ is a $\Sigma$-closed set of $\mathfrak{P}$ which is not $\Delta$-closed; and the closure of $\{P\}$ in $\mathfrak{B}_{\Delta}$ is not $\Sigma$-closed.

Lemma 2. If $I$ is an O-ideal, then $\mathfrak{B}(I)$ is closed in $\mathfrak{P}_{\Sigma}$ as well as in $\mathfrak{P}_{\Delta}$.

Proof. It is immediate that $\mathfrak{P}(I)$ is closed in $\mathfrak{P}_{\Sigma}$. To see that $\mathfrak{P}(I)$ is closed in $\mathfrak{B}_{\Delta}$, take $P_{o}$, a proper prime ideal, such that $P_{o} \subset \mathrm{U} \mathfrak{B}(I)$. Suppose $P_{o} D I$. Then there are elements $f, e \in I \backslash P_{o}$ such that $f e=f$. Therefore $f(1-e)=0 \in P_{o}$, so $1-e \in P_{o}$. Thus, there is $P \in \mathfrak{B}$ which contains $I$ and satisfies $1-e \in P$. But since $e \in I \subset P$, we have $1 \in P$, which is a contradiction, since $P$ is a proper ideal. Therefore $P_{o} \supset I$, and hence $\mathfrak{P}(I)$ is closed in $\mathfrak{P}_{\Delta}$.

Lemma 3. Let $\mathfrak{B}_{0}$ be a family of proper prime ideals containing a fixed maximal $O$-ideal $M$. If $\mathfrak{B}_{o}$ is a closed subset of $\mathfrak{B}_{\Sigma}$ as well as $\mathfrak{B}_{\Delta}$, then $\mathfrak{P}_{o}=\mathfrak{B}(M)$.

Proof. There exists exactly one maximal ideal $N$ such that $M \subset N$ (see a remark following 3.3 of [3] and 2.4 of [7]). Therefore every proper ideal containing $M$ is contained in $N$. Thus, $N \supset \cap \Re_{o}$ and $N \in \mathfrak{B}_{o}$, since $\mathfrak{B}_{o}$ is $\Sigma$-closed. Now if $P \in \mathfrak{B}(M)$ then $P \subset N \subset \cup \mathfrak{B}_{o}$, so $P \in \mathfrak{P}_{o}$, since $\mathfrak{B}_{o}$ is $\Delta$-closed. Hence, $\mathfrak{B}_{o}=\mathfrak{B}(M)$.

We now use several results from [7]: First, $C(X)$ is an $R$-ring (see 4.4, 4.5 (i) and (ii), and 5.3). Second, if $P$ is a prime ideal, then it is a prime-like ideal (see $\$ 2$ ), so Lemma 4.18 is applicable and yields the result that the set $L(P)=\{f: f e=f$ for some $e \in P\}$ is a maximal $O$-ideal of $C(X)$. Hence $L$ is a function from $\mathfrak{P}$ to $\mathfrak{M}$. Lemma 4.16 implies that $L$ is a continuous function from $\mathfrak{P}_{\Sigma}$ to $\mathfrak{M}_{\Delta}$. However, we shall sketch a direct proof of this fact. Let $\mathfrak{B}_{o}$ be a collection of proper prime ideals and suppose $P_{o} \in \mathfrak{P}$ is such that $P_{o} \supset \cap \mathfrak{B}_{o}$. Then

$$
L\left(P_{o}\right) \subset \cup L\left[\mathfrak{B}_{o}\right],
$$

for otherwise, there exist elements $f, e, e^{\prime} \in L\left(P_{o}\right) \backslash \cup L\left[\Re_{o}\right]$ such that 
$f e=f$ and $e e^{\prime}=e$. Therefore, $(1-e) e=0 \in \cap \Re_{o}$. Now $e \notin \cup \Re_{o}$, since $f \notin \cup L\left[\mathfrak{P}_{o}\right]$, so $1-e^{\prime} \in \cap \mathfrak{P}_{o} \subset P_{o}$. Since $e^{\prime} \in L\left(P_{o}\right) \subset P_{o}$, we have $1 \in P_{o}$. But $P_{o}$ is a proper ideal, so this is impossible. Hence $L\left(P_{o}\right) \subset \cup L\left[\mathfrak{P}_{o}\right]$ and we have proved

THEOREM 5. The function $L$ is a continuous function from $\mathfrak{P}_{\Sigma}$ to $\mathfrak{M}_{\Delta}$.

We can now prove

THEOREM 6. If $\mathfrak{B}_{0}$ is a family of proper prime ideals which is a closed subset of $\mathfrak{B}_{\Sigma}$ as well as $\mathfrak{P}_{\Delta}$, then $\cap \mathfrak{P}_{0}$ is an O-ideal.

Proof. Set $\mathfrak{M}_{o}=L\left[\mathfrak{P}_{0}\right]$. For any maximal $O$-ideal $M$, it is obvious that $L^{-1}(\{M\})=\mathfrak{B}(M)$, and therefore, by Lemma $2, L^{-1}(\{M\})$ is closed in both $\mathfrak{P}_{\Sigma}$ and $\mathfrak{P}_{\Delta}$. Thus $\mathfrak{P}_{0} \cap L^{-1}(\{M\})$ also has this property, so, by Lemma 3, $\mathfrak{B}_{0} \cap L^{-1}(\{M\})=\mathfrak{B}(M)$ for every $M \in \mathfrak{M}_{0}$. Therefore $M=\cap\left(\mathfrak{B}_{0} \cap L^{-1}(\{M\})\right)$, by Theorem 4 or $[4$, Theorem 1.4 ]. Hence $\cap \mathfrak{P}_{o}=\cap \mathfrak{M}_{o}$.

We now wish to know that $\mathfrak{M}_{0}$ is closed in $\mathfrak{M}_{\Delta}$. This is a consequence of the following facts: (i) $\mathfrak{P}_{\Sigma}$ is compact by [7, Remark 3.7], Corollary 2.4 and footnote 1 above; (ii) $\mathfrak{M}_{\Delta}$ is compact by [7, comments prior to 5.11]; (iii) $\mathfrak{P}_{o}$ is closed in $\mathfrak{P}_{\Sigma}$ by hypothesis and hence is compact; (iv) $\mathfrak{M}_{o}=L\left[\mathfrak{B}_{o}\right]$ is the continuous image of a compact set, so $\mathfrak{M}_{o}$ is $\Delta$-compact; (v) since $\mathfrak{M}_{\Delta}$ is a Hausdorff space [7, Corollary 4.9], $\mathfrak{M}_{o}$ is closed in $\mathfrak{M}_{\Delta}$.

Now the proof of the theorem can be completed. For by Theorem 3.9 of [7], the intersection of a family of maximal $O$-ideals which is closed in the dual Stone topology is an $O$-ideal. Hence, $\cap \mathfrak{\Re}_{o}=\cap \mathfrak{M}_{o}$ is an $O$-ideal.

Corollary 6.1. A necessary and sufficient condition that the intersection of a family of proper prime ideals $\mathfrak{B}_{0}$ be an O-ideal is that its closure $c_{2}\left(\mathfrak{P}_{0}\right)$ in $\mathfrak{P}_{\Sigma}$ be closed in $\mathfrak{P}_{\Delta}$.

Proof. Suppose $I=\cap \mathfrak{P}_{o}$ is an $O$-ideal. Now $c_{2}\left(\mathfrak{B}_{o}\right)=\mathfrak{B}(I)$, and by Lemma 2, this right hand set is closed in $\mathfrak{P}_{\Delta}$. Conversely, suppose $c_{2}\left(\mathfrak{P}_{o}\right)$ is a $\Delta$-closed set of $\mathfrak{P}$. It is certainly a $\Sigma$-closed set. Therefore, by the theorem, $\cap\left(c_{2}\left(\mathfrak{B}_{o}\right)\right)$ is an $O$-ideal. But it is easy to see that $\cap \Re_{o}=\cap\left(c_{2}\left(\mathfrak{P}_{o}\right)\right)$.

\section{REFERENCES}

1. R. L. Blair, Stone's topology for a binary relation, Duke Math. J. vol. 22 (1955) pp. 271-280.

2. L. Gillman, Rings with Hausdorff structure space, Fund. Math. vol. 45 (1957) pp. 1-16. 
3. L. Gillman and M. Henriksen, Concerning rings of continuous functions, Trans. Amer. Math. Soc. vol. 77 (1954) pp. 340-362.

4. - Rings of continuous functions in which every finitely generated ideal is principal, Trans. Amer. Math. Soc. vol. 82 (1956) pp. 366-391.

5. L. Gillman, M. Henriksen and M. Jerison, On a theorem of Gelfand and Kolmogoroff concerning maximal ideals in rings of continuous functions, Proc. Amer. Math. Soc. vol. 5 (1954) pp. 447-455.

6. E. Hewitt, Rings of real-valued continuous functions I, Trans. Amer. Math. Soc. vol. 64 (1948) pp. 45-99.

7. J. G. Horne, On the ideal structure of certain semirings and compactification of topological spaces, to appear in Trans. Amer. Math. Soc.

8. J. L. Kelley, General topology, New York, Van Nostrand, 1955.

9. N. H. McCoy, Rings and ideals, Mathematical Association of America, 1948.

10. A. N. Milgram, Multiplicative semigroups of continuous functions, Duke Math. J. vol. 16 (1949) pp. 377-383.

UNIVERSITY OF KENTUCKY

\section{CORRESPONDANCES SINGULIÈRES PAR PARALLÉLISME DES PLANS TANGENTS DES DEUX SURFACES}

\section{PAVEL DRĂGILĂ}

1. En étudiant la correspondance par parallélisme des plans tangents, dans les points homologues des deux surfaces, Peterson et ses continuateurs ont établi qu'en cas de cette correspondance, sur chaque surface il y a deux directions qui sont parallèles aux directions correspondantes sur l'autre surface. Ils croyaient avoir démontré aussi que ces directions déterminent toujours sur les deux surfaces des réseaux conjugués. Nous savons déjà que cette dernière assertion n'est pas éxacte.

Nous nous sommes proposé un nouveau problème, qui semble présenter d'importance: chercher s'il y en a des couples de deux surfaces en correspondance de parallélisme, de manière qu'elle éxiste sur une surface une seule direction, tangente à une courbe de coordonnées, qui soit parallèle à la direction correspondante sur l'autre surface, et, s'il est possible, de déterminer effectivement des telles surfaces.

Nous désignons la première surface par $S(x, y, z)$, la seconde par $\bar{S}(\bar{x}, \bar{y}, \bar{z})$ et les dérivées partielles $\partial r / \partial u, \partial r / \partial v, \partial^{2} r / \partial u \partial v \cdots$ par $r_{u}, r_{v}, r_{u v} \cdots$

Dans ce cas les coordonnées des deux surfaces doivent satisfaire l'un des deux systèmes:

Received by the editors August 15, 1957 and, in revised form, December 6, 1957. 